\title{
OPEN Bank vole prion protein extends the use of RT-QulC assays to detect prions in a range of inherited prion diseases
}

\author{
Tze How Mok, Akin Nihat, Connie Luk, Danielle Sequeira, Mark Batchelor, Simon Mead, \\ John Collinge \& Graham S. Jackson ${ }^{凶}$
}

The cerebrospinal fluid (CSF) real-time quaking-induced conversion assay (RT-QuIC) is an ultrasensitive prion amyloid seeding assay for diagnosis of sporadic Creutzfeldt-Jakob disease (CJD) but several prion strains remain unexplored or resistant to conversion with commonly used recombinant prion protein (rPrP) substrates. Here, bank vole (BV) rPrP was used to study seeding by a wide range of archived post-mortem human CSF samples from cases of sporadic, acquired and various inherited prion diseases in high throughput 384-well format. BV rPrP substrate yielded positive reactions in $70 / 79$ cases of sporadic CJD [Sensitivity $88.6 \%(95 \% \mathrm{Cl} 79.5-94.7 \%)$ ], 1/2 variant CJD samples, and 9/20 samples from various inherited prion diseases; 5/57 non-prion disease control CSFs had positive reactions, yielding an overall specificity of $91.2 \%(95 \% \mathrm{Cl} 80.1-97.1 \%)$. Despite limitations of using post-mortem samples and our results' discrepancy with other studies, we demonstrated for the first time that BV rPrP is susceptible to conversion by human CSF samples containing certain prion strains not previously responsive in conventional rPrPs, thus justifying further optimisation for wider diagnostic and prognostic use.

Prion disease represents a heterogeneous group of rare, transmissible and fatal neurodegenerative conditions affecting humans and other mammalian species, unified biologically by autocatalytic templated-misfolding of the host-encoded prion protein ( $\mathrm{PrP})$ into disease-associated form ${ }^{1}$. In spite of this singular mechanism of disease, the human conditions exhibit striking phenotypic diversity even within sporadic Creutzfeldt-Jakob disease (CJD), which comprises $80-85 \%$ of cases. Aside from the classical triad of rapid cognitive decline, gait ataxia and/or myoclonus, distinct clinical phenotypes such as the Heidenhain variant, ataxic variant (Brownell-Oppenheimer), corticobasal syndrome and sporadic fatal insomnia have been observed ${ }^{2}$. This diversity is hypothesised to be imparted by prion strains through the propagation in the brain of different pathological conformational states $^{1-3}$. There is evidence that this process is modified considerably by the polymorphic codon 129 of the prion protein gene $(P R N P)$. Strains can be inferred by laboratory assays including by western blot ${ }^{1,4,5}$. Highly-penetrant disease-causing PRNP mutations cause diseases historically classified into Gerstmann-Straussler-Scheinker (GSS) syndrome, fatal familial insomnia (FFI) and familial CJD (fCJD) ${ }^{6}$, although this fails to encapsulate the diversity of phenotypes resulting from octapeptide repeat insertions (OPRIs) and the more recently described peripheral systemic amyloidosis secondary to the PRNP Y163X mutation ${ }^{7,8}$.

Cerebrospinal fluid (CSF) examination has long been a key clinical investigation in individuals suspected to have sporadic CJD, in particular the detection of protein 14.3.3, raised S100B and exceedingly high total tau levels (typically $>1000 \mathrm{pg} / \mathrm{mL}$ ) are all considered to be highly supportive ${ }^{9-11}$. Though highly sensitive, the above are not seen exclusively in prion disease, and moreover are really surrogate markers of rapid axonal injury and gliosis; as such, histological examination endures in epidemiological case definitions as mandatory for definite diagnosis ${ }^{12}$.

More recently, real-time quaking-induced conversion (RT-QuIC) seeded by CSF has emerged as a rapid, sensitive $(>90 \%)$ and highly specific tool $(\approx 100 \%)$ for ante mortem diagnosis in $\mathrm{SCJD}^{13,14}$. The RT-QuIC exploits and accelerates the seeded amyloidosis of PrP through repeated cycles of mechanical agitation, coupled with Thioflavin $\mathrm{T}$ (ThT) fluorescence read-outs that can be monitored in real time. The typical RT-QuIC reaction mixture, composed vitally of a buffer, recombinant $\operatorname{PrP}(\mathrm{rPrP})$ and $\mathrm{ThT}$, has been successfully seeded by a variety of mammalian tissue and bodily fluids ${ }^{15-18}$. The efficiency and sensitivity of the reaction can be further enhanced 
by altering conditions such as raising temperature, $\mathrm{pH}$ and addition of sodium dodecyl sulphate (SDS) ${ }^{19}$, or by using different $r P r P$ constructs, giving rise to the so-called second generation IQ-CSF RT-QuIC ${ }^{20,21}$.

The extraordinary success of RT-QuIC in clinical diagnosis is at present restricted to SCJD and sCJD-like IPD phenotypes, mainly PRNP E200K. Save for a single study in CSF samples from patients with P102L and D178N mutations which successfully seeded RT-QuIC reactions using human $\mathrm{rPrP}^{22}$, the level of sensitivity has not been replicated in reactions seeded by CSF from GSS-causing IPD mutations (P102L, A117V, etc.) and variant CJD (vCJD) even with IQ-CSF RT-QuIC above. Other IPD mutations are simply too rarely encountered such that CSF may not be available for testing. Crucially, the lack of PrP sequence homology is expected to present a significant barrier to conversion, based on previous P102L and A117V human PrP transgenic mouse modelling experiments ${ }^{23,24}$. Thus, the expectation that a single $\mathrm{rPrP}$ type can serve as a 'universal acceptor' seemed improbable until some experiments showed efficient transmission of multiple prion strains in bank voles and in transgenic mice expressing bank vole $\mathrm{rPrP}(\mathrm{BV} \mathrm{rPrP})^{25,26}$, indicating a lack of both strain and species barriers. $\mathrm{BV} \mathrm{rPrP}$ as a 'universal acceptor' gained further traction when a series of RT-QuIC experiments seeded by brain homogenate $(\mathrm{BH})$ not only demonstrated seeding activity in variant CJD and GSS cases due to a number of PRNP mutations which were hitherto undetectable, but also in other mammalian species ${ }^{15}$.

Here, we have explored the universality of $\mathrm{BV} \mathrm{rPrP}$ as the substrate in RT-QuIC reactions seeded by CSF from a variety of human prion diseases and attempt to adapt the RT-QuIC technique to a 384-well microplate in place of the conventional 96-well format for higher throughput. The wealth of longitudinal clinical data gathered from the UK National Prion Monitoring Cohort (NPMC) since 2008 allowed us to then determine whether the RTQuIC seeding dose in post-mortem CSF correlates with the rate of functional decline in patients with sCJD ${ }^{27,28}$.

\section{Results}

Patients. A total of 101 archived post-mortem (PM) CSF samples from autopsy-proven prion disease cases were identified; this included SCJD $(\mathrm{n}=79)$, vCJD $(\mathrm{n}=2)$ and IPD $(\mathrm{n}=20)$ cases. The range of PRNP mutations in the IPD group encompassed P102L $(n=5)$, A117V $(n=2)$, Y163X $(n=1), D 178 N(n=2), E 196 K(n=1)$, E200K $(n=3), 4-O P R I ~(n=2)$ and 6-OPRI $(n=4)$. Control PM CSF samples were obtained from 49 anonymised autopsy-proven non-prion disease cases. An further set of 8 non-prion PM CSF samples were identified from the NPMC cohort from patients suspected to have sCJD clinically in life but PM examination revealed other causes of illnesses and deaths; PM CSF control samples amounted to 57 in total. A summary of patient demographics, including the PRNP codon 129 genotype where applicable and molecular strain type ${ }^{5}$ is available in Table 1 . The PM intervals (number of days between dates of death and post mortem examination) are available for all but 3 of our prion disease cases. The mean PM interval is $5.7 \pm 3.4$ days; that for the anonymised control cases is not known.

Optimisation of bank vole rPrP RT-QuIC assay. First, we compared the RT-QuIC assays using human (Hu) $\mathrm{rPrP}$ and $\mathrm{BV} \mathrm{rPrP}$ as substrates in the standard 96-well plate format at $45^{\circ} \mathrm{C}$, seeded by sCJD $(\mathrm{n}=13)$ and $\operatorname{vCJD}(\mathrm{n}=1)$ CSFs against control CSFs. Under the same conditions, RT-QuIC reactions using BV $\mathrm{rPrP}$ were positive for all sCJD CSFs (13/13), as opposed to 12/13 for reactions using Hu rPrP. Furthermore, the RT-QuIC reaction with $\mathrm{BV}$ rPrP was successfully seeded by vCJD but not using $\mathrm{Hu} \mathrm{rPrP}$, albeit with a longer lag phase and lower maximal relative fluorescence units (rfu) in the former compared to sCJD (Fig. 1).

Following that, RT-QuIC experiments were conducted with BV rPrP seeded by sCJD and vCJD cases against control CSFs in both final volumes of $50 \mu \mathrm{L}(\mathrm{CSF} 7.5 \mu \mathrm{L})$ and $75 \mu \mathrm{L}$ (CSF $11.25 \mu \mathrm{L}$ ) per well respectively in 384well plates to see if the reaction kinetics are replicated in smaller well volumes. Both final well volumes of $50 \mu \mathrm{L}$ and $75 \mu \mathrm{L}$ produced consistently positive reactions for both sCJD and vCJD, but at lower maximal rfu.

Finally, we then performed BV RT-QuIC reactions with $50 \mu \mathrm{L}$ final volumes at $50{ }^{\circ} \mathrm{C}$ in 384 -well plates. This showed enhanced maximal rfu for the positive reactions in wells seeded by SCJD and vCJD cases, replicating reaction kinetics seen in those conducted at final volumes of $100 \mu \mathrm{L}$ in 96 -well plates at $45^{\circ} \mathrm{C}$ (Fig. 2).

Bank vole rPrP RT-QuIC reactions seeded by PM CSFs. Once optimal conditions were determined $\left(50 \mu \mathrm{L}\right.$ final well volume $(7.5 \mu \mathrm{L} \mathrm{CSF})$ at $\left.50{ }^{\circ} \mathrm{C}\right)$, BV RT-QuIC reactions were run in 384 -well plates seeded by prion disease PM CSFs and non-prion non-neurological disease control PM CSFs (Fig. 3). In 79 cases of definite SCJD, 70 CSF samples were scored positive while 9 were negative; 5 out of 57 non-prion control PM CSF samples were scored positive solely based on exceeding the rfu threshold. This gave a sensitivity of $88.6 \%$ (95\% CI 79.5-94.7\%) and specificity of 91.2\% (95\% CI 80.7-97.1\%) for sCJD cases.

In the other subtypes, BV RT QuIC was positive in 1 of 2 vCJD, and 9/20 IPD [ 3 of 3 E200K, 1 of 5 P102L, 1 of 1 E196K, 1 of 2 D178N, 0 of 2 A117V, 0 of 1 Y163X, 0 of 2 4-OPRI and 3 of 4 6-OPRI cases (Table 2)].

Application of the Fisher's Exact Test to compare RT-QuIC results between sCJD and non-sCJD prion disease groups resulted in $p=0.001$.

Correlation of rate of decline in MRC scale score with prion seeding dose. Similar to Shi et al..$^{29}$, estimation of CSF prion seeding doses were derived from a standard calibration curve constructed from the lag phases (time to threshold rfu) of BV rPrP RT-QuIC reactions seeded by serial dilutions of sCJD BH (Fig. 4).

The rate of decline in individual MRC Scale scores from RT-QuIC positive CSF samples were calculated according to the mixed effects model described in Mead et al. ${ }^{28}$. Out of the 70 sCJD CSF samples that were scored positive, 61 had at least one MRC score recorded, but only 29 of 61 had sufficient data compiled to generate individual rates of MRC Scale score decline (percentage decline per day). In these 29 individuals, no significant correlation was found between prion seeding dose and functional decline $(r=-0.16, p=0.41)$ (Fig. 5A). This 


\begin{tabular}{|c|c|c|}
\hline & sCJD & vCJD \\
\hline Mean AAO (years) & 64 & 36 \\
\hline Mean duration (months) & 9.4 & 14.25 \\
\hline Male & 35 & 2 \\
\hline Female & 42 & 0 \\
\hline RT-QuIC positive & 70 & $1(\mathrm{MV})$ \\
\hline RT-QuIC negative & 9 & $1(\mathrm{MM})$ \\
\hline Codon 129MM & 36 & 1 \\
\hline Codon 129MV & 21 & 1 \\
\hline Codon 129VV & 13 & 0 \\
\hline Codon 129 unknown & 9 & 0 \\
\hline \multicolumn{3}{|c|}{ Molecular strain type (London classification) } \\
\hline 1 & 0 & \\
\hline 2 & 20 & \\
\hline 3 & 16 & \\
\hline 4 & 0 & 2 \\
\hline Not detected & 1 & \\
\hline Not available & 42 & \\
\hline \multicolumn{3}{|l|}{ Clinical syndrome } \\
\hline Classical & 26 & \\
\hline Ataxic & 15 & \\
\hline Cognitive & 21 & \\
\hline Psychiatric & 6 & \\
\hline Visual & 5 & \\
\hline Stroke-like & 0 & \\
\hline Sleep/thalamic & 2 & \\
\hline Corticobasal syndrome & 1 & \\
\hline Not available & 3 & \\
\hline
\end{tabular}

Table 1. Summary of patient demographics, $P R N P$ codon 129 and molecular strain type where available. This table summarises the relevant demographics from sporadic (sCJD) and variant CJD (vCJD) patients from whom CSF samples were obtained at post mortem which were included in the RT-QuIC assays. This covers mean age at onset (AAO), duration of illness in months, gender, RT-QuIC results, PRNP codon 129 genotype (some untested), molecular strain type (London Classification ${ }^{48}$ ) and clinical syndrome.

effect remained when patients were separated into their respective PRNP codon 129 genotypes $(M M(n=9)$, $\mathrm{r}=0.13, \mathrm{p}=0.73 ; \mathrm{MV}(\mathrm{n}=15), \mathrm{r}=-0.34, \mathrm{p}=0.21 ; \mathrm{VV}(\mathrm{n}=5), \mathrm{r}=-0.88, \mathrm{p}=0.05)$.

When the CSF prion seeding dose was compared against the total duration of disease available for all 61 patients instead of rate of MRC Scale score decline, a positive correlation was found $(r=0.44, p=0.0004)$, i.e. the longer the duration of disease, the higher the seeding dose (Fig. 5B). The correlation coefficients for the MM $(\mathrm{n}=28), \mathrm{MV}(\mathrm{n}=19)$ and VV $(\mathrm{n}=11)$ groups are $0.08(\mathrm{p}=0.68), 0.56(\mathrm{p}=0.02)$ and $-0.006(\mathrm{p}=0.98)$ respectively; the positive correlation in the MV group is largely accounted for by 2 outliers with extraordinarily long durations of disease (1944 days and 2401 days), over double that of any other patient.

\section{Discussion}

This study shows, for the first time in a sizeable sample set, that RT-QuIC reactions using BV rPrP as the conversion substrate can be successfully seeded by human prions in CSF, albeit autopsy-derived. Whilst the RT-QuIC had previously been adapted to the 384 -well format, it was confined to sheep scrapie BH seeds ${ }^{30}$; here, we show that this is similarly applicable to CSF containing human prions.

Interest in exploring the utility of BV rPrP in CSF RT-QuIC here followed a series of benchmark experiments carried out by Orrú et al. 2015 in which BV rPrP appeared to show potential as a 'universal' acceptor in a wide range (but small numbers of $n=2-4$ per species) of mammalian prion species ${ }^{15}$. Of particular interest were positive results from human BH samples of so-called GSS-causing mutations (P102L, F198S, A117V, H187R) with low molecular weight fragments on western blot, and D178N-FFI which had previously yielded negative results in reactions using hamster $\mathrm{rPrP}$ even with enhanced IQ-CSF conditions ${ }^{19}$. Furthermore, the failure of IQ-CSF RT-QuIC iterations (with either full length or truncated hamster $\mathrm{rPrP}$ ) to uniformly replicate their enhanced sensitivity in non-fCJD PRNP mutation samples emphasises this unmet clinical need for a universal acceptor ${ }^{18,20,21,31}$.

In the largest series of sCJD PM CSF samples in the literature tested with BV rPrP RT-QuIC to date, both the sensitivity and specificity of $88.6 \%$ (95\% CI 79.5-94.7\%) and 91.2\% (95\% CI 80.7-97.1\%) respectively in our study are below that reported in a number of recent large non-BV rPrPstudies (sensitivities 91-97.2\%, specificities $98-100 \%)$ in which the reactions are seeded by ante mortem CSF $14,18,20,21,31$. In our study, BV rPrP also 
A

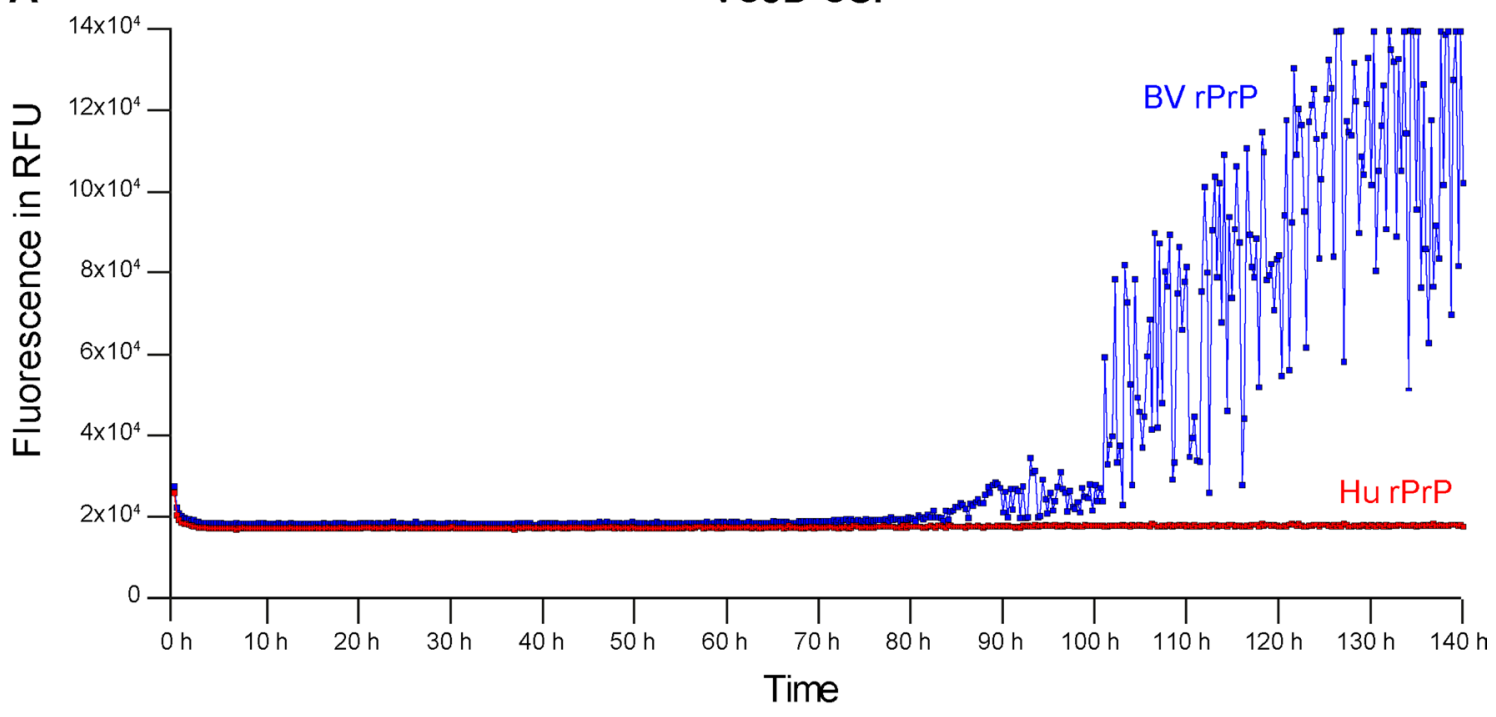

B VCJD vs SCJD vs Control CSFs with BV rPrP

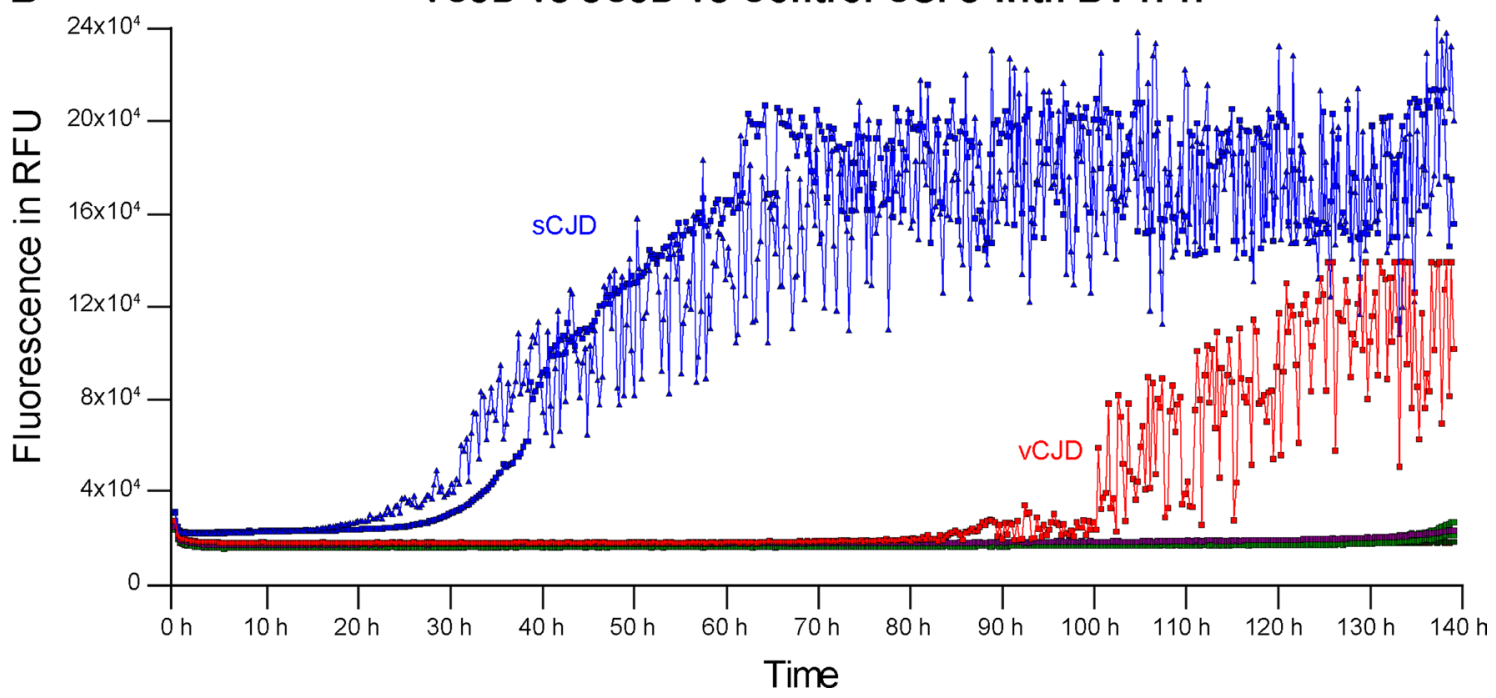

Figure 1. Comparisons between RT-QuIC reactions seeded by CSF samples from sCJD and vCJD cases, using either $\mathrm{Hu}$ rPrP or BV rPrP. Each curve is the mean with standard deviation of 4 replicate wells. (A) RT-QuIC reactions using $\mathrm{BV} \mathrm{rPrP}$ (blue) and $\mathrm{Hu} \mathrm{rPrP}$ (red) in 96-well format at $45^{\circ} \mathrm{C}$, seeded by vCJD CSF; $\mathrm{BV} \mathrm{rPrP}$ showed a positive result (2/4) but with a long lag phase while $\mathrm{Hu} r \mathrm{PrP}$ was negative (0/4). (B) Comparison of RT-QuIC reactions using BV rPrP, seeded by CSFs from sCJD (blue) and vCJD (red) cases at $45^{\circ} \mathrm{C}$; reactions seeded by sCJD CSFs showed the classic positive curves while that seeded by vCJD CSF replicated the delayed positive curve in (A).

appear to be successfully converted by other prion disease subtypes such as vCJD and a range of IPDs. While E200K, P102L and D178N CSF have been shown to seed RT-QuIC reactions in other studies using different $r P r P$ including full length $\mathrm{Hu} \mathrm{rPrP}$, full length hamster $\mathrm{PPrP}$, truncated Syrian hamster rPrP (90-231) and hamstersheep chimeric $\mathrm{rPrP}^{20-22,31,32}$, our study here showed for first time that CSF from cases of 6-OPRI IPD can seed RT-QuIC reactions.

The lower sensitivity and specificity of CSF BV rPrP RT-QuIC in SCJD, and particularly its even lower sensitivity across IPD subtypes appear to be at variance with the findings in Orrù et al. ${ }^{15}$, and suggest that perhaps BV rPrP may not be a completely "universal acceptor" of prion strains. Moreover, we are aware of a selection of 19 CSF samples (from a small collection of 22 sCJD and 4 fCJD (E200K) ante mortem CSF) tested under BV rPrP RT-QuIC conditions similar to Orrù et al. ${ }^{15}$ which returned an even lower sensitivity relative to our study of $52.6 \%{ }^{33}$. Possible explanations for our discrepancies may lie in the differential sample matrix and RT-QuIC conditions, and the presence of 'conversion' barriers, or a combination thereof.

Firstly, the use of PM CSF in this pilot study is relatively unconventional as well-established CSF RT-QuIC protocols in use are honed chiefly for ante mortem CSF which has a different fluid matrix to PM CSF, and PM CSF is expected to have lower seeding activity compared to BH previously tested with BV rPrP RT-QuIC. However, given the large volumes of PM CSF that can be collected relative to ante mortem CSF, we judged it to 

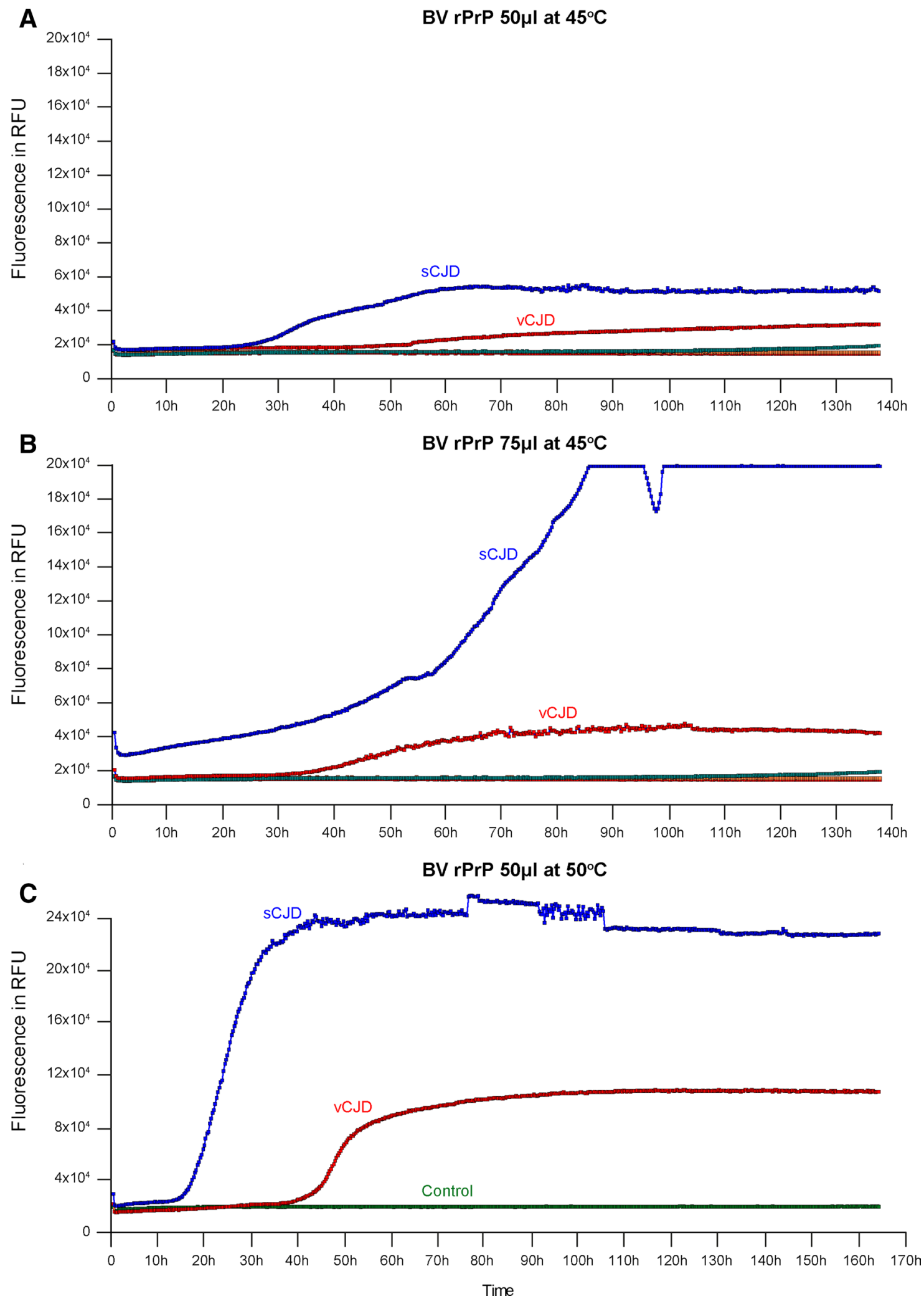

Figure 2. Comparisons of reaction volumes in RT-QuIC reactions using BV rPrP in 384-well format. Reactions seeded by CSF samples from sCJD and vCJD at (A) total volume of $50 \mu \mathrm{L}$ at $45^{\circ} \mathrm{C}$, (B) total volume of $75 \mu \mathrm{L}$ at $45^{\circ} \mathrm{C}$ and $(\mathbf{C})$ total volume of $50 \mu \mathrm{L}$ at $50^{\circ} \mathrm{C}$. Each curve is the mean with standard deviation of 4 replicate wells. This series of experiments showed that positive results can be replicated at lower reaction and analyte volumes; raising the temperature to $50{ }^{\circ} \mathrm{C}$ improved the efficiency of the reaction by reducing the lag phase and by producing a higher maximal $\mathrm{rfu}$. 
A

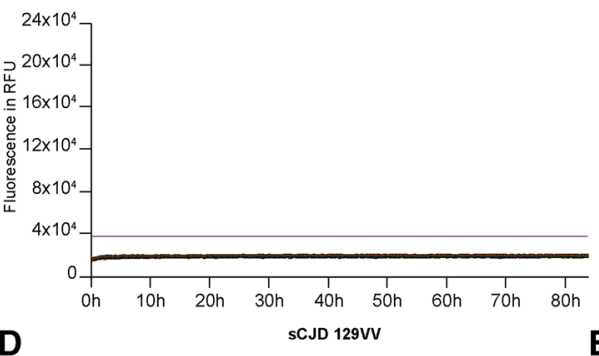

D

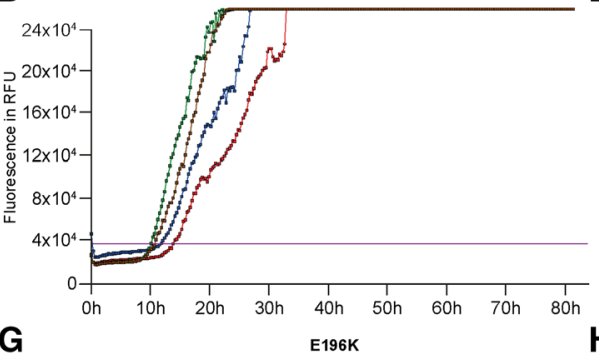

B
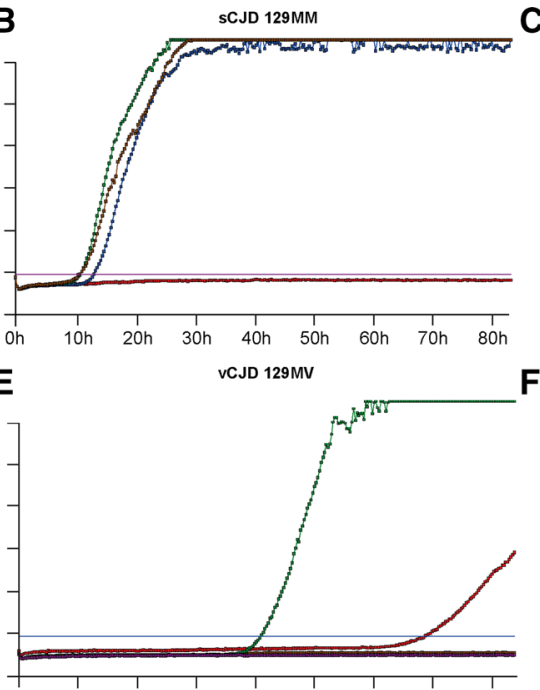

C
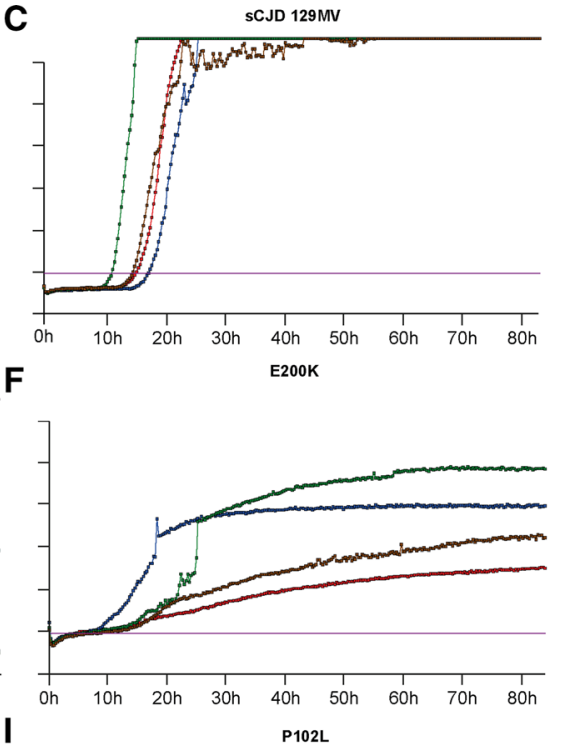
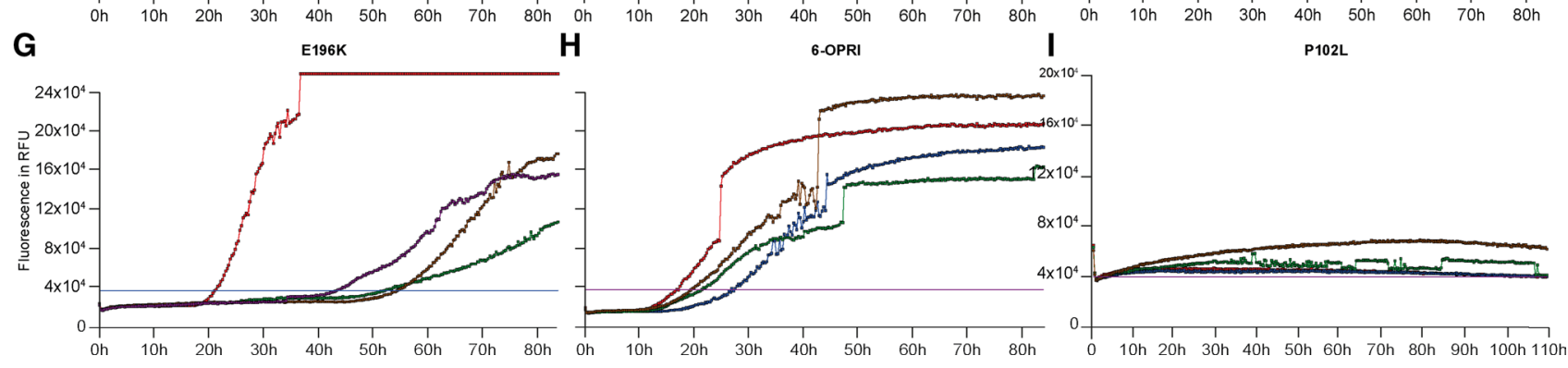

Figure 3. Sample traces of RT-QuIC reactions using BV rPrP in 384-well format. Reaction volumes of $50 \mu \mathrm{L}$ at $50{ }^{\circ} \mathrm{C}$ seeded by CSF from (A) Alzheimer's Disease (AD), (B) sCJD 129MM, (C) sCJD 129MV, (D) sCJD 129VV, (E) vCJD 129MV, (F) E200K, (G) E196K, (H) 6-OPRI and (I) P102L. Positive results were seen in sCJD with different $P R N P$ codon 129 genotypes, vCJD and a range of IPD mutations, while the reaction seeded by AD CSF remained negative throughout. Individual coloured signal curves in each panel represent the signal curves of each well (of which there are 4) of the corresponding prion species/type indicated.

\begin{tabular}{|c|c|c|c|c|}
\hline & RT-QuIC positive & RT-QuIC negative & Sensitivity \% & Specificity\% \\
\hline sCJD & 70 & 9 & 88.6 & \\
\hline vCJD & 1 & 1 & 50 & \\
\hline P102L & 1 & 4 & 20 & \\
\hline $\mathrm{A} 117 \mathrm{~V}$ & 0 & 2 & 0 & \\
\hline Y163X & 0 & 1 & 0 & \\
\hline D178N & 1 & 1 & 50 & \\
\hline E196K & 1 & 0 & 100 & \\
\hline E200K & 3 & 3 & 100 & \\
\hline 4-OPRI & 0 & 2 & 0 & \\
\hline 6-OPRI & 3 & 1 & 75 & \\
\hline \multicolumn{3}{|l|}{ All PRNP mutations } & 45 & \\
\hline Non-prion disease controls & 5 & 52 & & 91.2 \\
\hline
\end{tabular}

Table 2. Post mortem CSF RT-QuIC results according to types of prion disease (including individual diseasecausing PRNP mutations), and non-prion disease controls. This table shows that breakdown of CSF RT-QuIC results according to type of prion disease including sporadic CJD (sCJD), variant CJD (vCJD) and individuals PRNP mutations (P102L, A117V, Y163X, D178N, E196K, E200K, 4-OPRI, 6-OPRI). The sensitivity and specificity of bank vole rPrP RT-QuIC for sCJD is 88.6\% (95\% CI 79.5-94.7\%) and 91.2\% (95\% CI 80.7-97.1\%) respectively, as opposed to the overall sensitivity of all PRNP mutations of $45 \%$. 


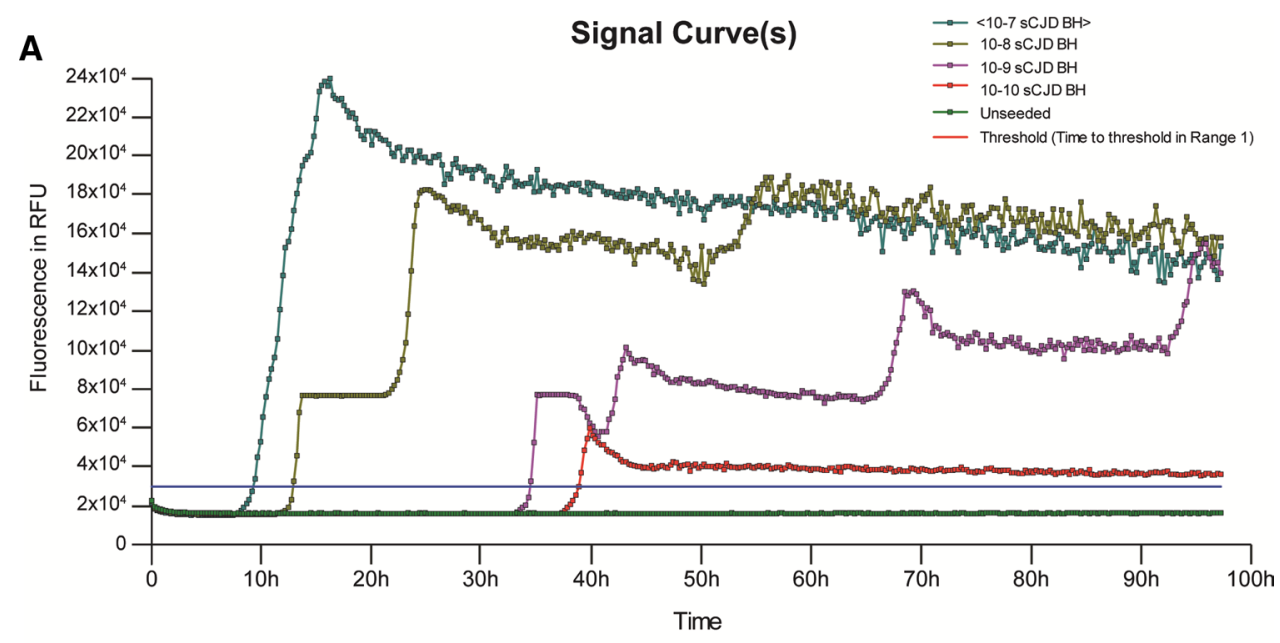

B

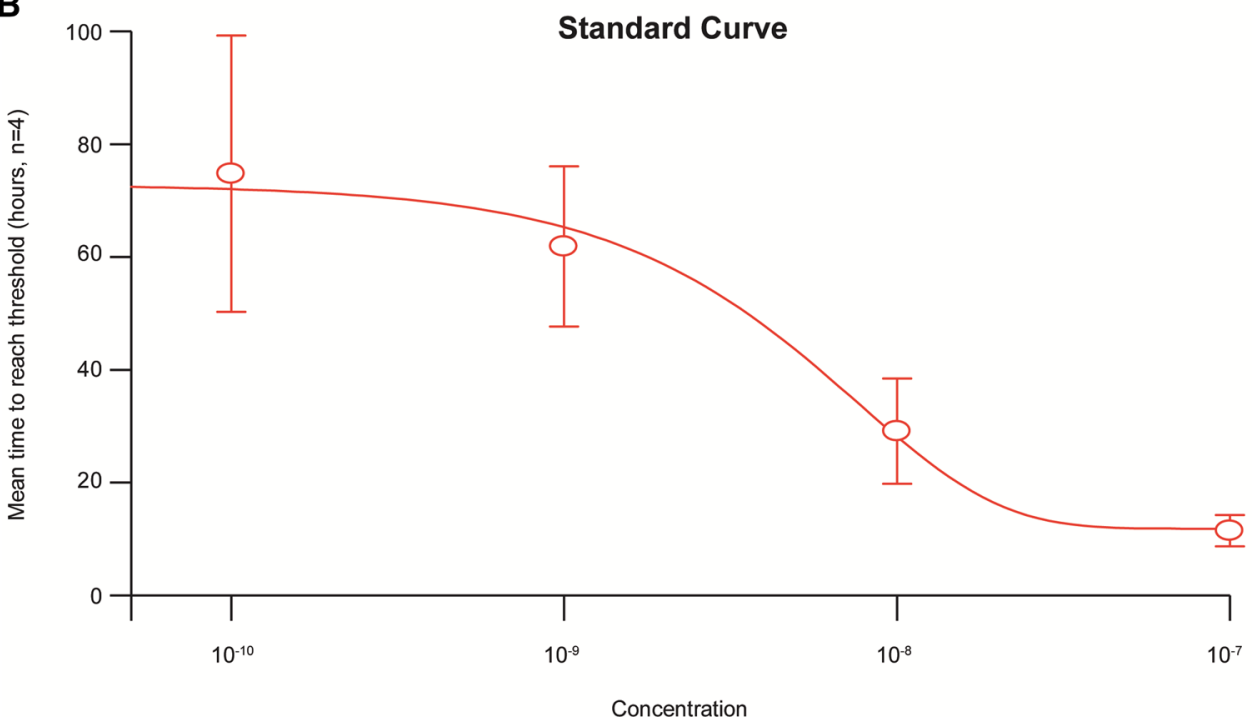

Figure 4. Estimation of CSF prion seeding doses. RT-QuIC reactions seeded by serial dilutions of sCJD $129 \mathrm{MM}$ brain homogenates at $50^{\circ} \mathrm{C}$; each curve representing the average rfu readings across replicate wells (A), and dose response curve for estimation of prion seeding doses based on time to threshold lag phases (B). Estimation of CSF prion seeding doses were derived from the standard calibration curve (B) constructed from the lag phases (time to threshold rfu) of RT-QuIC reactions with BV rPrP seeded by serial dilutions of sCJD BH in (A). Data points in (B) represent the mean of 4 wells with standard errors of the mean.

be an appropriate biofluid medium to conduct an exploratory study in a reasonably large sample set. Key CSF composition factors identified to have profound effects on RT-QuIC reaction kinetics include red cell count (haemoglobin concentration), white cell count and protein level. The haem concentration in CSF samples with red cell counts exceeding $1250 \times 10^{6} / \mathrm{L}$ may quench the fluorescent signal sufficiently to return false negative results ${ }^{34}$; on the other hand, protein levels greater than $1.0 \mathrm{~g} / \mathrm{L}$ and raised white cell count beyond $10 \times 10^{6} / \mathrm{L}$ are liable to cause false positive misinterpretations due to high and fluctuating baseline rfu measurements ${ }^{35}$. It is recognised that significant physicochemical changes take place in death, even in closed compartment fluids like $\mathrm{CSF}^{36}$; broadly speaking, leakage of impurities into PM CSF which follows cessation of active transport mechanisms that maintain the blood-brain barrier such as cell debris and proteins can inhibit amyloid seeding assays $^{37-39}$. More specifically, CSF total protein level is known to increase significantly by about 20 times (mean $8.37 \mathrm{~g} / \mathrm{L}$ ) in agonal death durations greater than $6 \mathrm{~h}$ such as that observed in prion disease ${ }^{40}$, and perhaps further increased with longer death to autopsy intervals ${ }^{41}$. As for PM CSF white cell count, it increases progressively with post mortem interval, and autopsy studies indicate that CSF cell count would have exceeded $10 \times 10^{6} / \mathrm{L}$ by $10 \mathrm{~h}$ post mortem ${ }^{42}$.

Cell counts and routine biochemistry were not performed on our PM CSF sample set in clinical laboratories for biohazard concerns, although visually pink or red samples indicating significant red cell contamination were discarded from analyses. In spite of this, it remains likely that the PM CSF samples used here (with mean PM 

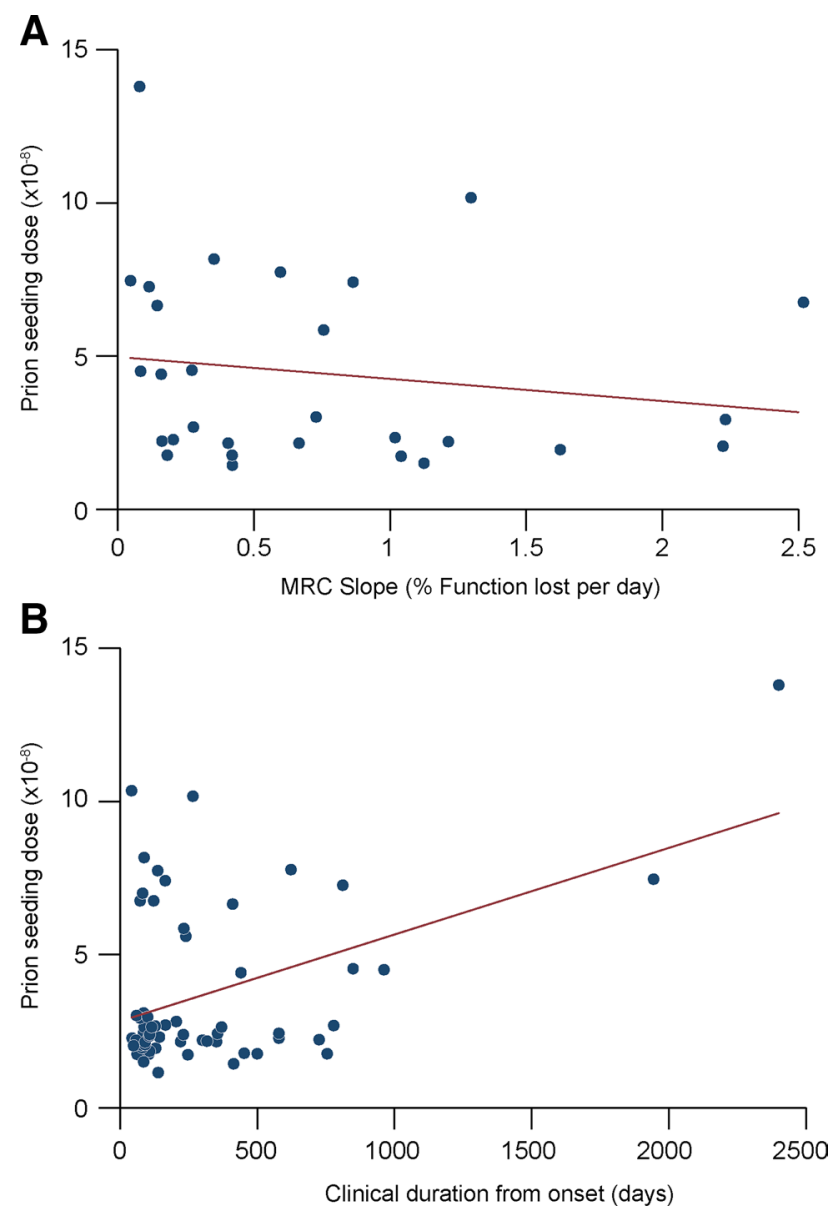

Figure 5. Correlation of CSF prion seeding doses with functional decline and duration of illness. Correlation of the MRC Scale slope (percentage decline in function per day) with prion seeding dose by RT-QuIC (A), and Correlation of the clinical duration from symptom onset with prion seeding dose by RT-QuIC (B). No correlation was seen with rate of functional decline (A) but a positive correlation was found $(r=0.44, p=0.0004)$ with total duration of illness. However the latter positive correlation is dependent on 2 extreme outliers with long durations of illness.

intervals of 5.6 days) contain total protein levels and white cell counts (or its debris) greatly in excess of $1.0 \mathrm{~g} / \mathrm{L}$ and $10 \times 10^{6} / \mathrm{L}$ respectively. Indeed, all 5 of our positive control PM CSF samples were scored as such due to high and fluctuating baseline. These 5 reaction kinetics and curves were distinct from conventional positive results, and arguably should have been considered 'negative' from a qualitative perspective. These anonymised control samples were obtained from a different institution where only the ages of death and gender were provided; these patients died of non-neurological causes (and thus presumably non-prion), some of which involve trauma, but the precise cause of death for each individual was not provided. As a result, we did not have the resources to age- and gender-match all cases for this exploratory study.

Aside from the different seed matrices between Orrù et al..$^{15}$ and our study, other differences are summarised in Table 3. Overall, it is difficult to discern if a singular factor within these could account for the discrepancy, notwithstanding the expected lower seed concentration in CSF compared to BH. However limited experience with ante mortem CSF BV rPrP RT-QuIC to date does suggest that reaction efficiency for BV rPrP RT-QuIC is not as readily replicated between seed matrices compared to IQ-CSF using truncated hamster $\mathrm{rPrP}^{19,20}$. One may be tempted to raise the reaction temperature but BV rPrP RT-QuIC has tended to spontaneously fibrillise between the 60-70th hour even at a $42{ }^{\circ} \mathrm{C}^{15}$.

One other key consideration is the possibility that there may still exist "conversion" barrier(s) with BV rPrP RT-QuIC if differential seeding activity and matricial compositional factors are insufficient to explain the result discrepancy. The collated literature does suggest that CSF from PRNP mutations with a fCJD phenotype, in particular the E200K mutation, can easily seed RT-QuIC reactions, but less so in others which produce the GSS or FFI phenotypes ${ }^{20,21,31}$. What this is down to is not entirely clear but this disparity seemed to be resolved by moving towards $\mathrm{rPrP}$ sequence homology where RT-QuIC conversion rates between different IPDs were shown to be comparable by using full length $\mathrm{Hu} \mathrm{rPrP}^{22}$; Sano et al. in 2013 achieved positive RT-QuIC reactions from E200K (18/22), P102L (18/20) and D178N-FFI (10/12) CSF samples by using full length Hu rPrP ${ }^{22}$. However it ought to be pointed out that the reaction solution composition, temperature and microplate reader is significantly different to the RT-QuIC protocols employed by us and Orrù et al. ${ }^{15}$. Mouse modelling studies with GSS-related 


\begin{tabular}{|c|c|c|c|}
\hline RT-QuIC conditions & Our study & Orrù et al. ${ }^{15}$ & Vallabh et al. ${ }^{33}$ \\
\hline \multicolumn{4}{|c|}{ Reaction mix components/well } \\
\hline Phosphate buffer (pH 7.4) & $10 \mathrm{mM}$ & $10 \mathrm{mM}$ & $10 \mathrm{mM}$ \\
\hline $\mathrm{NaCl}$ & $300 \mathrm{mM}$ & $300 \mathrm{mM}$ & $300 \mathrm{mM}$ \\
\hline EDTA & $10 \mu \mathrm{M}$ & $1 \mathrm{mM}$ & $1 \mathrm{mM}$ \\
\hline ThT & $10 \mu \mathrm{M}$ & $10 \mu \mathrm{M}$ & $10 \mu \mathrm{M}$ \\
\hline SDS & None & $0.001 \%$ & $0.001 \%$ \\
\hline $\mathrm{rPrP}$ & $0.1 \mathrm{mg} / \mathrm{mL}$ & $0.1 \mathrm{mg} / \mathrm{mL}$ & $0.1 \mathrm{mg} / \mathrm{mL}$ \\
\hline $\mathrm{H}_{2} \mathrm{O}$ & \multicolumn{3}{|c|}{ As required to make up respective well volume } \\
\hline Seed type & PM CSF & $\mathrm{BH}$ & AM CSF \\
\hline See volume per well & $7.5 \mu \mathrm{L}$ & $2 \mu \mathrm{L}$ & $20 \mu \mathrm{L}$ \\
\hline Total volume/well & $50 \mu \mathrm{L}$ & $100 \mu \mathrm{L}$ & $100 \mu \mathrm{L}$ \\
\hline Microplate format & 384-well & 96-well & 96-well \\
\hline $\mathrm{BV}$ rPrP construct & Cleaved his-tagged & Non his-tagged & Non his-tagged \\
\hline Reaction temperature & $50^{\circ} \mathrm{C}$ & $42^{\circ} \mathrm{C}$ & $42^{\circ} \mathrm{C}$ \\
\hline
\end{tabular}

Table 3. Comparison of RT-QuIC key conditions used in this study against those of Orrù et al..$^{15}$ and Vallabh et al. ${ }^{33}$. $\mathrm{NaCl}$ sodium chloride, EDTA ethylenediaminetetraacetic acid, ThT thioflavin T, SDS sodium dodecyl sulphate, $r P r P$ recombinant prion protein, $P M C S F$ post mortem cerebrospinal fluid, AM CSF ante mortem cerebrospinal fluid, $\mathrm{BH}$ brain homogenate, his-tagged histidine-tagged.

P102L and A117V prions where prions derived from the respective mutant protein can only be transmitted to transgenic mice expressing the corresponding mutant human PrP, and not to wild type mice or mice expressing wild type human $\operatorname{PrP}^{23,24}$, may give this further traction. It is thus possible that prions from certain IPD mutations may require mutant human $\mathrm{rPrP}$ to allow conversion in $\mathrm{RT}-\mathrm{QuIC}$ reactions.

The RT-QuIC assay is largely perceived as a "reporting" assay in which the outcome is binary, though attempts have been made at probing RT-QuIC kinetics and products to elucidate the prion seeding dose in test samples. RT-QuIC end-point dilution remains the most accurate way of measuring seeding-competent PrP conformers in a test sample in place of animal bioassay ${ }^{38}$, but the quantitative method proposed by Shi et al. (similar to that employed here) based on correlating dose against reaction lag times on a calibrated curve, appears to promise efficiency in terms of time and spatial requirements ${ }^{29}$. CSF is routinely only drawn once for diagnostic purposes, and regular CSF sampling at intervals during the illness is hard to justify both clinically and ethically. On the expectation that PM CSF contains the "final and maximal" CSF prion seeding dose, we explored if the extrapolated dose scorrelates with the rates of functional decline in these patients, measured by the percentage decline per day of the validated MRC Scale score obtained in life ${ }^{27,28}$. In this study, no clear correlation was found between seeding dose and rate of MRC Scale score (functional) decline. A moderate positive correlation was counterintuitively observed between prion seeding dose and total illness duration across all patients; however, once segregated into codon 129 genotypes this relationship remained statistically significant only for patients with the MV genotype, and could be accounted for by a mere 2 outliers with extremely long durations of illness. This is perhaps unsurprising as it is not precisely known whether a single or multiple species of permissible conformational strains of prions are present within any single biofluid sample and which ones are capable of seeding RT-QuIC reactions ${ }^{43}$. Moreover, the two-phase kinetic model of prion propagation distinguishes infectious prions and neurotoxic PrP species whose production is uncoupled ${ }^{44,45}$. In other words, the abundance of the toxic species mediating functional decline may not be reflected in RT-QuIC seeding competency, and vice versa. Also, rate of decline data was only available from about a third of sCJD patients in the sample set, and as such the numbers may be too small to draw any firm conclusions at the present time.

Our pilot study demonstrated that BV rPrP RT-QuIC can be successfully seeded by PM CSF from a relatively large and diverse sample set derived from prion disease patients, and that it can be adapted to the 384-well format to increase throughput and conserve limited CSF volumes. Significant difference remains between its efficiency for SCJD and IPD groups, and as such is at variance with its previously shown "universality" with prion species ${ }^{15}$. This however does not necessarily invalidate BV rPrP's potential as a "universal acceptor" of prions as the disparities may merely reflect differences in the matrices of test samples (and hence their seeding doses) and/or in the RT-QuIC conditions used. Nevertheless, we believe our results justifies further optimisation of this assay for wider diagnostic and prognostic use.

\section{Methods}

Research governance, clinical data, post mortem examination and sample preparation. CSF and brain tissue used in this study were obtained at post mortem from patients who were suspected to have prion disease while alive, and subsequently archived at the Medical Research Council Prion Unit at UCL. The study was performed with approval from the London-Queen Square Research Ethics Committee (Reference: 03/ N022). Fully informed consent was obtained, and samples procured and used in accordance with the approved study and relevant national legislation and guidelines. 
The majority of these patients were enrolled either into the National Prion Monitoring Cohort (NPMC) or the MRC PRION-1 Trial ${ }^{46}$ the autopsies were largely performed at the National Hospital for Neurology \& Neurosurgery on Queen Square or less frequently in local hospitals, both in rooms designated for high-risk autopsies. Full research consent was obtained for all samples used in this study; Scotland A Research Ethics Committee (NPMC) or the Eastern Research Ethics Committee (PRION-1). Prion disease patients enrolled into the NPMC and PRION-1 Trial had regular clinical assessments and their functional status recorded as the Medical Research Council Prion Disease Rating Scale (MRC Scale x/20) over time ${ }^{27,46}$.

At each autopsy, CSF was aspirated by pipette from the pontine cistern by access through the skull base; this is achieved sequentially by skull removal, separation of dura, lifting of frontal and temporal lobes, and separations of the optic chiasm from the skull base and pituitary gland from pituitary stalk respectively. All CSF samples were stored at $-80^{\circ} \mathrm{C}$ prior to analysis. CSF from neuropathologically confirmed prion cases were obtained from MRC Prion Unit at UCL. Fully anonymised non-neurological control post-mortem (PM) CSF samples were obtained from Dr Esiri at the Oxford Brain Bank.

Brain homogenates $(\mathrm{BH} ; 10 \% \mathrm{w} / \mathrm{v})$ were prepared as previously described ${ }^{6}$ and stored at $-80^{\circ} \mathrm{C}$. Aliquots of $10 \% \mathrm{w} / \mathrm{v} \mathrm{BH}$ were thawed at room temperature freshly for each RT-QuIC assay. The BHs were diluted serially in artificial CSF $\left(150 \mathrm{mM} \mathrm{NaCl}, 2 \mathrm{Mm} \mathrm{CaCl}_{2}, 1.2 \mathrm{mM} \mathrm{MgCl}_{2}, 1.5 \mathrm{mM} \mathrm{K}_{2} \mathrm{HPO}_{4}\right.$ and $10 \mathrm{mM}$ glucose, $\mathrm{pH}$ 7.3) in the quantitative RT-QuIC assay.

Expression and purification of full length human (Hu rPrP) and bank vole (BV rPrP) recombinant PrP. Hu rPrP (aa residues 23-231; with methionine at codon 129; accession M13899) and BV rPrP (aa residues 23-231; with methionine at codon 129; accession AF367624) were prepared according to Jackson et al. 1999 with minor modifications ${ }^{47}$. Briefly, the DNA sequences encoding either $\mathrm{rPrP}$ in pTrcHisB were transformed into BL21 (DE3). BL21 cultures were grown in LB medium in the presence of $100 \mu \mathrm{g} / \mathrm{ml} \mathrm{Ampicil-}$ lin. Expression of either rPrP was then induced using $1 \mathrm{mM}$ IPTG, and purified from inclusion bodies under denaturing conditions using Nickle superflow resin with an AKTA Pure (GE Healthcare Life Sciences). Subsequently, the $\mathrm{rPrP}$ was eluted from the column using an imidazole gradient, and the eluted material was extensively dialysed against $20 \mathrm{mM}$ Bis Tris $\mathrm{pH}$ 6.5. The $\mathrm{rPrP}$ Histidine tag was cleaved with the addition of $2.5 \mathrm{mM}$ $\mathrm{CaCl}_{2}$ and 50U Thrombin (VWR). Following this, the $\mathrm{rPrP}$ was run through a second NiNTA on the AKTA Pure to remove the Histidine tag, and dialysed against $10 \mathrm{mM}$ Bis Tris $\mathrm{pH}$ 6.5. The concentration of $\mathrm{rPrP}$ was determined by absorption at $280 \mathrm{~nm}$ with molar extinction coefficients of $61,880 \mathrm{M}^{-1} \mathrm{~cm}^{-1}$ and $56,667 \mathrm{M}^{-1} \mathrm{~cm}^{-1}$ for $\mathrm{BV} \mathrm{rPrP}$ and $\mathrm{Hu} \mathrm{rPrP}$ respectively. Recombinant $\mathrm{PrP}$ concentration was adjusted to $0.2-0.5 \mathrm{mg} / \mathrm{mL}$ by the addition of buffer. Aliquots were stored at $-80^{\circ} \mathrm{C}$ until use. Prior to usage, aliquot(s) of $\mathrm{rPrP}$ was dialysed into $20 \mathrm{mM}$ Sodium phosphate buffer ( $\mathrm{pH} 5.8$ ), filtered ( $0.22 \mu \mathrm{m}$ syringe).

RT-QuIC analysis of CSF and BH. The RT-QuIC reaction buffer was composed of $10 \mathrm{mM}$ phosphate buffer ( $\mathrm{pH}$ 7.4), $300 \mathrm{mM} \mathrm{NaCl}, 0.1 \mathrm{mg} / \mathrm{mL} \mathrm{rPrP}, 10 \mu \mathrm{M}$ Thioflavin T (ThT), and $10 \mu \mathrm{M}$ ethylenediaminetetraacetic acid (EDTA). Reactions were prepared in either 96-well or 384-well optical clear-bottomed plates (Nalgene Nunc International 265301; 242764). For 96-well plates, each well contained either $98 \mu \mathrm{L}$ of reaction buffer seeded by $2 \mu \mathrm{L}$ of $\mathrm{BH}$ or $85 \mu \mathrm{L}$ of reaction buffer seeded by $15 \mu \mathrm{L}$ CSF, bringing the total volume in each well up to $100 \mu \mathrm{L}$. The proportion of the components, and hence its concentrations were maintained when applied to different volumes in 384-well plate experiments e.g. $42.5 \mu \mathrm{L}$ reaction buffer seeded by $7.5 \mu \mathrm{L}$ CSF (total $50 \mu \mathrm{L}$ ). Each CSF sample was run in quadruplicate, allowing for 5 negative controls (PM non-prion CSF) and 5 positive controls (definite sCJD CSFs).

Thereafter, the plates were sealed and incubated in a BMG OPTIMA FLUOstar plate reader at either $45^{\circ} \mathrm{C}$ or $50^{\circ} \mathrm{C}$ for $90-120 \mathrm{~h}$ with cycles of intermittent shaking. Each cycle consisted of $60 \mathrm{~s}$ of double orbital shaking at $700 \mathrm{rpm}$ followed by $60 \mathrm{~s}$ rest, with ThT fluorescence measurements ( $450 \mathrm{~nm}$ excitation; $480 \mathrm{~nm}$ emission) taken every $2 \mathrm{~min}$. The plate reader measures ThT fluorescence in relative fluorescence units (rfu) with a maximum rfu at 260,000. A reaction was considered positive if the average rfu in 2 or more wells exceeded a threshold rfu defined as the average rfu from 5 negative controls plus 5 standard deviations, and within $90 \mathrm{~h}$ of start time.

Statistical analysis. The sensitivity and specificity were calculated for SCJD and IPD groups, and statistical significance between groups was determined by Fisher's Exact Test (Stata, v15.1). Mixed effect linear modelling of MRC Scale scores was undertaken with Stata, v15.1.

Received: 10 August 2020; Accepted: 17 February 2021

Published online: 04 March 2021

\section{References}

1. Collinge, J. Prion diseases of humans and animals: Their causes and molecular basis. Annu. Rev. Neurosci. 24, 519-550 (2001).

2. Parchi, P. et al. Classification of sporadic Creutzfeldt-Jakob disease based on molecular and phenotypic analysis of 300 subjects. Ann. Neurol. 46, 224-233 (1999).

3. Collinge, J., Sidle, K. C. L., Meads, J., Ironside, J. \& Hill, A. F. Molecular analysis of prion strain variation and the aetiology of 'new variant' CJD. Nature 383, 685-690 (1996).

4. Hill, A. F. et al. Distinct glycoform ratios of protease resistant prion protein associated with PRNP point mutations. Brain J. Neurol. 129, 676-685 (2006).

5. Wadsworth, J. D. F. et al. Molecular diagnosis of human prion disease. Methods Mol. Biol. Clifton 459, 197-227 (2008).

6. Mead, S. Prion disease genetics. Eur. J. Hum. Genet. EJHG 14, 273-281 (2006).

7. Mead, S. \& Reilly, M. M. A new prion disease: relationship with central and peripheral amyloidoses. Nat. Rev. Neurol. 11, 90-97 (2015). 
8. Mead, S. et al. A novel prion disease associated with diarrhea and autonomic neuropathy. N. Engl. J. Med. 369, 1904-1914 (2013).

9. Thompson, A. G. B. \& Mead, S. H. Review: Fluid biomarkers in the human prion diseases. Mol. Cell. Neurosci. https://doi. org/10.1016/j.mcn.2018.12.003 (2018).

10. Hsich, G., Kenney, K., Gibbs, C. J., Lee, K. H. \& Harrington, M. G. The 14-3-3 brain protein in cerebrospinal fluid as a marker for transmissible spongiform encephalopathies. N. Engl. J. Med. 335, 924-930 (1996).

11. Muayqil, T., Gronseth, G. \& Camicioli, R. Evidence-based guideline: Diagnostic accuracy of CSF 14-3-3 protein in sporadic Creutzfeldt-Jakob disease: Report of the guideline development subcommittee of the American Academy of Neurology. Neurology 79, 1499-1506 (2012).

12. Diagnosis and Testing|CJD. https://www.cjd.ed.ac.uk/surveillance/diagnosis-and-testing.

13. Atarashi, R. et al. Ultrasensitive human prion detection in cerebrospinal fluid by real-time quaking-induced conversion. Nat. Med. 17, 175-178 (2011).

14. McGuire, L. I. et al. Real time quaking-induced conversion analysis of cerebrospinal fluid in sporadic Creutzfeldt-Jakob disease. Ann. Neurol. 72, 278-285 (2012).

15. Orrú, C. D. et al. Bank vole prion protein as an apparently universal substrate for RT-QuIC-based detection and discrimination of prion strains. PLoS Pathog. 11, e1004983 (2015).

16. Orrú, C. D. et al. Rapid and sensitive RT-QuIC detection of human Creutzfeldt-Jakob disease using cerebrospinal fluid. $m B i o$ 6, $1(2015)$.

17. Orrú, C. D. et al. Prion seeding activity and infectivity in skin samples from patients with sporadic Creutzfeldt-Jakob disease. Sci. Transl. Med. 9, 1 (2017).

18. Bongianni, M. et al. Diagnosis of human prion disease using real-time quaking-induced conversion testing of olfactory mucosa and cerebrospinal fluid samples. JAMA Neurol. 74, 155-162 (2017).

19. Orrú, C. D. et al. Factors that improve RT-QuIC detection of prion seeding activity. Viruses 8, 140 (2016).

20. Groveman, B. R. et al. Extended and direct evaluation of RT-QuIC assays for Creutzfeldt-Jakob disease diagnosis. Ann. Clin. Transl. Neurol. 4, 139-144 (2017).

21. Franceschini, A. et al. High diagnostic value of second generation CSF RT-QuIC across the wide spectrum of CJD prions. Sci. Rep. 7, 10655 (2017).

22. Sano, K. et al. Early detection of abnormal prion protein in genetic human prion diseases now possible using real-time QUIC assay. PLoS ONE 8, e54915 (2013).

23. Asante, E. A. et al. Transmission properties of human PrP 102L prions challenge the relevance of mouse models of GSS. PLoS Pathog. 11, e1004953 (2015).

24. Asante, E. A. et al. Inherited prion disease $\mathrm{A} 117 \mathrm{~V}$ is not simply a proteinopathy but produces prions transmissible to transgenic mice expressing homologous prion protein. PLoS Pathog. 9, e1003643 (2013).

25. Nonno, R. et al. Efficient transmission and characterization of Creutzfeldt-Jakob disease strains in bank voles. PLoS Pathog. 2, e12 (2006).

26. Watts, J. C. et al. Evidence that bank vole PrP is a universal acceptor for prions. PLoS Pathog. 10, e1003990 (2014).

27. Thompson, A. G. B. et al. The Medical Research Council prion disease rating scale: a new outcome measure for prion disease therapeutic trials developed and validated using systematic observational studies. Brain J. Neurol. 136, 1116-1127 (2013).

28. Mead, S. et al. Clinical trial simulations based on genetic stratification and the natural history of a functional outcome measure in Creutzfeldt-Jakob disease. JAMA Neurol. 73, 447 (2016).

29. Shi, S., Mitteregger-Kretzschmar, G., Giese, A. \& Kretzschmar, H. A. Establishing quantitative real-time quaking-induced conversion (qRT-QuIC) for highly sensitive detection and quantification of PrPSc in prion-infected tissues. Acta Neuropathol. Commun. 1, 44 (2013).

30. Metrick, M. A. et al. Million-fold sensitivity enhancement in proteopathic seed amplification assays for biospecimens by Hofmeister ion comparisons. Proc. Natl. Acad. Sci. 116(46), 23029-23039 (2019).

31. Foutz, A. et al. Diagnostic and prognostic value of human prion detection in cerebrospinal fluid. Ann. Neurol. 81, 79-92 (2017).

32. Cramm, M. et al. Characteristic CSF prion seeding efficiency in humans with prion diseases. Mol. Neurobiol. 51, 396-405 (2015).

33. Vallabh, S. M. et al. Cerebrospinal fluid and plasma biomarkers in individuals at risk for genetic prion disease. BMC Med. 18, 140 (2020).

34. Cramm, M. et al. Stability and reproducibility underscore utility of RT-QuIC for diagnosis of Creutzfeldt-Jakob disease. Mol. Neurobiol. 53, 1896-1904 (2016).

35. Green, A. J. E. RT-QuIC: A new test for sporadic CJD. Pract. Neurol. 19, 49-55 (2019).

36. Peyron, P.-A., Lehmann, S., Delaby, C., Baccino, E. \& Hirtz, C. Biochemical markers of time since death in cerebrospinal fluid: A first step towards "Forensomics". Crit. Rev. Clin. Lab. Sci. 56, 274-286 (2019).

37. Colby, D. W. et al. Prion detection by an amyloid seeding assay. Proc. Natl. Acad. Sci. 104, 20914-20919 (2007).

38. Wilham, J. M. et al. Rapid end-point quantitation of prion seeding activity with sensitivity comparable to bioassays. PLoS Pathog. 6, e1001217 (2010).

39. Horiuchi, M., Priola, S. A., Chabry, J. \& Caughey, B. Interactions between heterologous forms of prion protein: Binding, inhibition of conversion, and species barriers. Proc. Natl. Acad. Sci. USA. 97, 5836-5841 (2000).

40. Mangin, P., Lugnier, A.-A., Chaumont, A.-J., Offner, M. \& Grucker, M. Forensic significance of postmortem estimation of the blood cerebrospinal fluid barrier permeability. Forensic Sci. Int. 22, 143-149 (1983).

41. Osuna, E., Perez-Carceles, M. D., Luna, A. \& Pounder, D. J. Efficacy of cerebro-spinal fluid biochemistry in the diagnosis of brain insult. Forensic Sci. Int. 52, 193-198 (1992).

42. Wyler, D., Marty, W. \& Bir, W. Correlation between the post-mortem cell content of cerebrospinal fluid and time of death. Int. J. Legal Med. 106, 194-199 (1994).

43. Collinge, J. \& Clarke, A. R. A general model of prion strains and their pathogenicity. Science 318, 930-936 (2007).

44. Sandberg, M. K., Al-Doujaily, H., Sharps, B., Clarke, A. R. \& Collinge, J. Prion propagation and toxicity in vivo occur in two distinct mechanistic phases. Nature 470, 540-542 (2011).

45. Sandberg, M. K. et al. Prion neuropathology follows the accumulation of alternate prion protein isoforms after infective titre has peaked. Nat. Commun. 5, 4347 (2014).

46. Collinge, J. et al. Safety and efficacy of quinacrine in human prion disease (PRION-1 study): A patient-preference trial. Lancet Neurol. 8, 334-344 (2009).

47. Jackson, G. S. et al. Multiple folding pathways for heterologously expressed human prion protein. Biochim. Biophys. Acta BBA 1431, 1-13 (1999).

48. Hill, A. F. et al. Molecular classification of sporadic Creutzfeldt-Jakob disease. Brain J. Neurol. 126, 1333-1346 (2003).

\section{Acknowledgements}

We would like to thank the patients and their families, and the contribution of numerous local physicians to the patients' care and investigation. This work was funded by the UK Medical Research Council. The clinical research activities of the National Prion Clinic are supported by the National Institute of Health Research's (NIHR) Biomedical Research Centre at University College London Hospitals NHS Foundation Trust. THM is supported 
by a Fellowship award from Alzheimer's Society, UK (Grant Number 341 (AS-CTF-16b-007)). AN is supported by a Fellowship award from the UK Medical Research Council. Both THM and AN are also supported by CJD Support Network UK Research Support Grants. SM and JC are NIHR Senior Investigators.

\section{Author contributions}

Conceived and designed the experiments: S.M., J.C. and G.S.J. Performed the experiments: T.H.M., C.L. and M.B. Analysed the data: T.H.M., C.L. and A.N. Contributed materials: M.B., S.M. Wrote the manuscript: T.H.M., S.M. and G.S.J. All authors critically reviewed the manuscript.

\section{Competing interests}

The authors declare no competing interests.

\section{Additional information}

Correspondence and requests for materials should be addressed to G.S.J.

Reprints and permissions information is available at www.nature.com/reprints.

Publisher's note Springer Nature remains neutral with regard to jurisdictional claims in published maps and institutional affiliations.

(c) (i) Open Access This article is licensed under a Creative Commons Attribution 4.0 International

License, which permits use, sharing, adaptation, distribution and reproduction in any medium or format, as long as you give appropriate credit to the original author(s) and the source, provide a link to the Creative Commons licence, and indicate if changes were made. The images or other third party material in this article are included in the article's Creative Commons licence, unless indicated otherwise in a credit line to the material. If material is not included in the article's Creative Commons licence and your intended use is not permitted by statutory regulation or exceeds the permitted use, you will need to obtain permission directly from the copyright holder. To view a copy of this licence, visit http://creativecommons.org/licenses/by/4.0/.

(C) The Author(s) 2021 